Published in final edited form as:

Nurs Outlook. 2015 ; 63(2): 154-161. doi:10.1016/j.outlook.2014.07.005.

\title{
Chronic Disease Self-Management: A Hybrid Concept Analysis
}

\author{
Wendy R. Miller, PhD, RN, CCRN [Assistant Professor], \\ Indiana University School of Nursing \\ Sue Lasiter, PhD, RN [Assistant Professor], \\ Indiana University School of Nursing \\ Rebecca Bartlett Ellis, PhD, RN [Assistant Professor], and \\ Indiana University School of Nursing \\ Janice M. Buelow, PhD, RN, FAAN [Vice President of Programs and Research] \\ Epilepsy Foundation of America
}

\section{Abstract}

Background-Chronic diseases require chronic disease self-management (CDSM). Existing CDSM interventions, while improving outcomes, often do not lead to long-lasting effects. To render existing and new CDSM interventions more effective, an exploration of the concept of CDSM from both the literature and patient perspectives is needed.

Purpose-To describe the current conceptualization of CDSM in the literature, identify potential inadequacies in this conceptualization based on a comparison of literature- and patient-based CDSM descriptions, and to offer a more comprehensive definition of CDSM.

Method-A hybrid concept analysis was completed.

Discussion-In the literature, CDSM is defined as behaviors influenced by individual characteristics. Patients in the fieldwork phase discussed aspects of CDSM not well-represented in the literature.

Conclusions-CDSM is a complex process involving behaviors at multiple levels of a person's environment. Pilot work to develop and test CDSM interventions based on both individual and external characteristics is needed.

\section{Introduction}

Chronic disease is the central health care problem in the United States (U.S.) and beyond. Nearly one out of two Americans suffer from at least one chronic disease, and chronic diseases contribute to the majority of U.S. health care costs. Further, chronic diseases are

(C) 2014 Mosby, Inc. All rights reserved.

Publisher's Disclaimer: This is a PDF file of an unedited manuscript that has been accepted for publication. As a service to our customers we are providing this early version of the manuscript. The manuscript will undergo copyediting, typesetting, and review of the resulting proof before it is published in its final citable form. Please note that during the production process errors may be discovered which could affect the content, and all legal disclaimers that apply to the journal pertain. 
responsible for downturns in quality of life $(\mathrm{QoL})$, and are the leading cause of disability and death in the U.S. (Centers for Disease Control and Prevention, 2013).

Chronic diseases often require extensive lifestyle changes and consistent attention from both the person who has the disease and the care provider. Typically incurable and characterized by exacerbations and remissions, chronic diseases must be managed throughout a lifetime. Together, healthcare providers, patients, and families are charged with managing the day-today treatments, symptoms, and lifestyle modifications associated with chronic diseases, which impact all aspects of one's life, making the management of the disease complex. When patients and families manage the complexity of disease characteristics, symptoms, and restructure an acceptable lifestyle, patients and their families greatly contribute to better outcomes (Unger \& Buelow, 2009).

Due to the ubiquity of chronic diseases and the costliness associated with them, there has been a clear need for the development of interventions targeting chronic disease selfmanagement (CDSM); many such interventions exist (Barlow, Sturt, \& Hearnshaw, 2002) and are structured to prepare patients to manage diseases to improve outcomes. While outcomes such as health status, QoL, and adherence have been improved by CDSM interventions based on behavior change theories, these changes are often short-lived. Though some interventions have demonstrated effects on outcomes up to four years postintervention (Lorig et al., 1993), these effects are generally smaller than at six months to one year post-intervention (Barlow et al., 2008), and have been found to disappear after five years (Caplin \& Creer, 2001). Clearly, for persons with chronic diseases that last a lifetime, achieving desired effects for only four years is insufficient.

Researchers must discover ways in which to achieve more comprehensive and longer lasting effects of CDSM by building upon existing interventions. Before such interventions can be devised, a more comprehensive conceptualization of CDSM is needed. While prior research has demonstrated that individual characteristics affect important outcomes and are amenable to intervention, due to fading effects of these interventions, other aspects important to CDSM may be missed. The purpose of this paper is to describe the current conceptualization of CDSM in the literature, to identify potential inadequacies in the current conceptualization of the concept, and to offer a more comprehensive definition of CDSM. This definition will be derived via a hybrid concept analysis of CDSM. A hybrid concept analysis allows for the inclusion of actual patient experiences in defining a concept; thus, this method allows for a more patient-centered definition.

\section{Background}

As pointed out by Lorig and Holman (2003), CDSM has been conceptualized in a variety of ways over the past four decades. However, in nursing literature, CDSM has been represented primarily as a verb and refers to the behaviors that persons use to manage the disease and its associated effects. That is, what people $d o$ constitutes their self-management (Lorig \& Holman, 2003). These actions may be in the form of direct, observable behaviors, cognitive strategies, or decisions (Lorig \& Holman, 2003). Similarly, Unger and Buelow (2009) found that the way in which CDSM has been conceptualized in nursing and medical 
literature can best be described as actions persons with chronic disease take concerning medication and treatment compliance, safety, event management, and lifestyle management.

Researchers have conducted investigations to specify and further explicate the selfmanagement behaviors used by those with chronic diseases. Corbin and Strauss (1988), for example, found that CDSM behaviors occur within three realms: medical management, adopting new behaviors or life roles, and dealing with associated emotions. Later, based on 25 years of research with persons self-managing chronic diseases, Lorig and colleagues (2001) identified five core self-management behaviors that occur as features of the selfmanagement of most chronic diseases: problem-solving, decision-making, resource utilization, forming of a relationship with a provider, and taking action. These five core behaviors are executed in the three realms outlined by Corbin and Strauss (1988). Lorig and Holman (2003) also noted that an additional core behavior of self-management is selftailoring, which refers to persons with chronic disease using the afore-mentioned core behaviors based on personal evaluation of their own needs and not necessarily their healthcare providers' evaluation of their needs.

Self-management is also neither an endpoint nor an outcome, but a process that affects and leads to outcomes (Holman \& Lorig, 2004; Osborne, Wilson, Lorig, \& McColl, 2007; Ryan $\&$ Sawin, 2009). However, as articulated by Clark (2003), changing self-management behaviors is useless if those behaviors do not improve outcomes such as QoL or perceived or actual health status. While self-management affects important outcomes, selfmanagement is not an outcome (Ryan \& Sawin, 2009) in and of itself. Self-management, then, is a portion of the means to an end, but not the end.

Internal, individual characteristics such as knowledge, (Clark, 2003; Ryan \& Sawin, 2009), self-efficacy (Lorig et al., 2001; Marks et al., 2005a; Marks et al., 2005b), and selfregulation (Clark, 2003; Ryan \& Sawin, 2009) have been shown to affect CDSM. Thus, investigators have developed interventions aimed at altering these characteristics, and in so doing, alter CDSM outcomes. Some of these interventions have successfully improved CDSM outcomes, the most notable of which is Lorig and colleagues' (2001) Chronic Disease Self-Management Program, which is based on social-cognitive theory (Bandura, 1986). In one study, this intervention was delivered to over 900 persons with arthritis, diabetes, chronic lung disease, and stroke. As part of the intervention, participants learned about cognitive symptom management, exercise program adoption, fatigue and sleep management, medication and community resource utilization, emotions (fear, anger, and depression) management, problem solving, and decision making; they also received training in how to effectively communicate with healthcare providers (Lorig et al., 2001). At two years post-intervention, significant and desirable changes were noted in healthcare resource utilization (frequency of emergency room visits), health status (in the form of health distress), and experiences of symptom fatigue. Perceived functional disability was improved at one year, but this result did not persist to the two-year follow-up assessment (Lorig et al., 2001). A Cochrane review of CDSM interventions revealed similar findings-while theorybased CDSM interventions can positively impact outcomes, these effects are typically not long lasting (Coster \& Norman, 2009). 
The noticeable absence of long-lasting effects of CDSM interventions that target individual characteristics such as self-efficacy may be due, at least in part, to the failure of such interventions to target external characteristics in a person's life. Researchers have begun to consider the use of other, more comprehensive theories on which to build CDSM interventions. Moore and colleagues (2013), for example, have used the socio-ecological theory to develop CDSM interventions. This theory is grounded in complexity and considers interrelations between personal and environmental factors and how they affect behavior. Interventions targeting both internal and external factors of persons engaging in CDSM may be more robust. Moore and colleagues (2013) have reported positive results regarding initial outcomes from their interventions, though long-term effects have yet to be measured.

Given that most existing CDSM interventions have been primarily focused on persons' internal characteristics, and have not been able to demonstrate long-term effects, there is a need to expand the conceptualization of CDSM. The generation of such a definition can serve as the foundation for the development of CDSM interventions with longer-lasting effects.

\section{Methods}

A hybrid concept analysis was employed. This method utilizes both theoretical analysis of literature and empirical observation to define a concept (Rodgers \& Knafl, 1993). We chose the hybrid concept analysis method because its inclusion of patient perspectives allowed for the generation of a more patient-centered definition of the concept. The method consists of three phases: theory, fieldwork, and analysis. The theoretical phase involves an analysis of the literature. Its purpose is to capture the overall essence of a concept and how it is defined, used, and measured in the literature. The fieldwork phase further refines the concept definition via the collection of qualitative data from patients. In the analytic phase, data from the fieldwork phase are compared with findings from the theoretical phase to produce a refined definition of the concept that is supported by both literature and patient perspectives.

As part of the theoretical phase, a review of the literature was undertaken to identify the conceptual and operational definitions of CDSM used in research and identify antecedents, consequences, and outcomes of CDSM. Any reference to a model of care or theoretical framework identified in the studies was documented, as well as references to the complexity of self-care management. Two independent searches were performed to identify relevant articles using the databases PubMed, Ovid Medline, and CINAHL and limited to years 2000 to 2013. Keywords were "chronic illness" AND "self-management" AND "self care," "adherence," "compliance" and "interventions." Articles were limited to "adults only." The search yielded 316 papers and with duplicates removed, leaving 263 papers for review.

These titles and abstracts were reviewed independently by two team members and selected if the focus of the article was on CDSM. Finally, thirty-seven articles were excluded because they focused on practice interventions to improve provider efficiency and provider outcomes in care delivery, rather than being patient-focused. Four researchers undertook a comprehensive review of the remaining 226 papers. A reference table was constructed that displayed all articles that met inclusion criteria and places for the four researchers to enter 
data into the table about antecedents, outcomes, and complexity that were referenced within the articles.

In the analytic phase, qualitative data generated from a prior qualitative study of adult epilepsy patients' descriptions of their self-management experiences (Miller, Bakas, \& Buelow, 2014) were re-analyzed via content analysis to meet the purpose of the current study; codes were generated, and tested against all data (Sandelowski, 1995). Themes regarding participants' explanations of their CDSM emerged. Finally, these themes were compared to findings rendered during the theoretical phase. Similarities and differences between the two sets of findings were noted.

\section{Findings: Theoretical Phase Theory-based Perspectives}

Of the 226 papers that met inclusion criteria, 111 papers included a specific theoretical perspective that guided or influenced the approach to the self-management studies. Selfefficacy Theory (Bandura, 1997), Chronic Care Model (Bodenheimer, 2002; Wagner, 1997), and Patient Activation (Hibbard, 2004) were the most frequently used theoretical frameworks. These, and other theoretical frameworks less frequently used, can be found in Table 1.

\section{Definitions of Self-management}

Overall, 111 papers included a clear theoretical definition of CDSM. Authors who did not provide a definition of CDSM proceeded to freely use the term with the assumption of a universally understood definition. Authors who provided a theoretical definition often did so without an operational definition or left the reader to determine how CDSM could be operationalized. Self-management was operationalized in 181 papers using various instruments. Expected behaviors such as daily exercise, blood glucose monitoring, or following a prescribed diet were measured and reported in 90 studies. These behaviors were specified or prescribed by a healthcare provider and instruments were used to quantify the frequency of the expected behavior or to measure the impact of the behavior relative to a physiological parameter. Researchers in 87 papers used disease status or physiological parameters such as HgbA1c, BMI, body weight, cholesterol, forced vital capacity, or number of seizures to operationalize the effectiveness of or success of client selfmanagement.

In the case of a chronic disease, measures of knowledge about a specific disease process and how to manage symptoms was the objective/outcome of interest in four papers. Knowing about the disease and what to do about a symptom, should it occur, was not necessarily predictive of action on the part of the patient to self-manage.

\section{Antecedents}

Antecedents were mentioned in 82 papers, either intentionally or as an indirect way of describing populations that could use self-management skills. Of note is that, in all articles reviewed, the presence of a chronic condition was implied as an antecedent for engaging in 
self-management. Authors considered self-efficacy $(n=64)$, disease knowledge $(n=44)$, social support $(n=16)$, health beliefs $(n=4)$, motivation $(n=4)$, and coping $(n=2)$ as antecedents to CDSM engagement.

\section{Consequences}

In the articles reviewed, consequences of CDSM included a variety of outcomes, including disease status/severity (such as symptom frequency and severity, number of exacerbations, physiologic parameters) $(n=131)$, treatment adherence $(n=107)$, health care resource utilization ( $\mathrm{n}=104)$, functional ability $(\mathrm{n}=96)$, and $\mathrm{QoL}(\mathrm{n}=92)$. Functional ability and QoL were considered distal outcomes.

\section{Complexity}

Overall, authors of the articles reviewed gave little attention to the complex nature of CDSM in conceptually and operationally defining the concept. For the purposes of this study, complexity was considered part of authors' definitions of CDSM if explicitly stated in manuscripts reviewed, or if authors alluded to complexity by including external factors (such as relationships with others or social environment) as integral to a patient's CDSM. The complex nature of CDSM was explicitly mentioned in only 21 articles. In 11 articles, CDSM was noted to be complex and structured, and seven authors noted that outside influences such as social relationships or environmental factors influence a person's ability to engage in CDSM. In three articles, however, the complexity of CDSM was considered and discussed at length. In these three articles, authors discussed the concept of CDSM using the socio-ecological model (Zebrowitz-McArthur \& Baron, 1983).

\section{Working Definition of CDSM}

Based on findings from the theoretical phase, a working, literature-based definition of CDSM has been generated: CDSM consists of behaviors in which those with chronic diseases engage in order to achieve medication/treatment adherence and manage symptoms and exacerbations; the outcomes of CDSM are the attainment of some degree of quality of life and disease control. Behaviors engaged in as part of CDSM are primarily related to a person's self-efficacy to execute those behaviors.

\section{Findings: Fieldwork Phase}

The transcripts of five persons with epilepsy were reviewed. Three were female, and two were male. The average age of participants was 61. Four participants were Caucasian, while one was African American. Three themes emerged: engaging in behaviors, going from exacerbation to chronicity and back again, and using a system.

\section{Engaging in behaviors}

In describing their CDSM, all five participants spoke of engaging in a variety of behaviors to self-manage their condition. That is, they described CDSM as an active process. These behaviors were related to treatment adherence, symptom management/prevention, and social/work situations. One participant explained, "I take care of the side effects of my meds by making sure I take a nap every single day. It's part of what I have to do to help keep my 
life normal." Another noted, "I can't lose my job...I can't retire yet...so I have to adjust my medicine schedule to make sure I am alert at work."

\section{Going from chronicity to exacerbation and back again}

Four of the five participants spoke of having to manage going from managing their condition in a chronic state, and then suddenly changing their CDSM to deal with an exacerbation. One participant explained, "I get on auto pilot. I'm doing great, going along and then I have a seizure. Now I can't drive, can't work for a while, you know. It's terrible. It's like a roller coaster." Another explained, "It's always hard...when I have gone in the hospital and then I come home and everyone treats me kind of like a baby. I feel like that's a really hard part of what I have to do, to go up and down, back and forth. It never stays the same because I might be doing perfect and then 'boom!' everything goes up in smoke...and I hate it."

\section{Using a system}

All five participants described managing their condition within their environment. In all cases, this environment involved managing epilepsy in the midst of interpersonal and other relationships. Some reported that close family members and friends share in the CDSM. "I don't do this alone," reported one. "It's both of us. Me and her. We manage it together." Others described how their CDSM had become embedded in work or volunteer activities. "Exercise really helps me with the fatigue from my [medication]. So I've volunteered at the animal shelter for years...and instead of just sitting at the desk I actually get out and walk the dogs. So volunteering is something I would do anyway....and now I can help myself, take care of that fatigue, while I'm there. So it's not me thinking how am I going to have time to get a walk in. I just do it while I'm there. I take every opportunity I have all day long to try to make myself feel better...epilepsy is in every part of my life."

\section{Findings: Analytic Phase}

In comparing the conceptualization of CDSM as seen in the literature with that described by patients actually self-managing a chronic disease, both similarities and differences were noted. The most prominent finding that appeared in both sets of data was that CDSM is a behavioral process. However, in the literature, these behaviors are most frequently defined as those related to treatment adherence; the fieldwork phase revealed that patients engage in behaviors far beyond those related to treatment-including those related to symptom and daily life management. Thus, the current behavioral conceptualization of CDSM in the literature may be incomplete.

As was seen in a small portion of the literature, participants involved in the fieldwork phase depicted CDSM as a systems-based process, and felt that their condition and its management did not exist in isolation, but rather had become part of nearly every aspect of their lives. As described earlier, the literature depicts CDSM as a process primarily based on individual, personal characteristics. However, our findings from the fieldwork phase indicate that multiple levels of a person's environment, including relationships with others and work and volunteer activities, do indeed become part of a person's CDSM. 
Not found during the theoretical phase was the notion that patients with chronic diseases must alter their CDSM based on sudden and acute exacerbation phases of their usually stable, chronic condition. The literature thus does not include discussion of this real and vital part of patients' experiences of CDSM.

\section{Toward a More Complete Definition of CDSM}

Based on this hybrid concept analysis, we offer a new, more complete definition of CDSM-one that is representative of both theoretical and actual patient perspectives. Chronic disease self-management is a fluid, iterative process during which patients incorporate multidimensional strategies that meet their self-identified needs to cope with chronic disease within the context of their daily living. Strategies are multidimensional because they require the individual to incorporate intrapersonal, interpersonal, and environmental systems (see Table 2) to maximize wellness. Successful management of both functioning in day-to-day life along with management of chronic illness requires the individual to continually monitor health and functional status and take appropriate actions during acute phases.

The management of both functioning in daily life and managing chronic illness requires patients to continually evaluate their perceived health status (which comprises how they feel emotionally and physically and how they are able to function on a daily basis) and implement unique variety of behaviors to manage their medications/treatments, safety, seizures, physical and emotional comfort, functional status, and other factors depending on their disease status.

Self-managed chronic disease is not a steady state of illness or disease but constitutes a continuous ebb and flow of symptoms of illness that may interfere with functional daily life (Mishel, 1999; Fulton \& Baird, 2014). As a patient experiences acute episodes within a chronic disease state, continued successful self-management requires reframing and readjusting one's understanding of the dimensions of the illness and devise new ways to selfmanage; thus, self-management is an iterative process. The challenge for people living with a chronic illness is that the complexity of life is an integral part of a complex system that can often get in the way of managing a condition. Understanding that CDSM is not a linear process that begins with an illness and ends with an outcome, rather it occurs within a "complex system" - a system for which CDSM emerges from the interaction of the many parts that comprise each layer of the system and for which may not be evident in the parts alone. Therefore, the traditional two-dimensional linear view of self-management is insufficient in understanding and describing CDSM.

Successful self-management allows the individual to function as an individual in his or her daily living and within the interpersonal and family environment, as a partner within the healthcare environment, and within the social community environment while also implementing strategies to maximize their wellness within the context of illness and chronic disease. We all live in a complex world and what is important is that we begin to look for patterns that emerge from these non-linear relationships. As clinicians, we need to know how to look for patterns from each person's complex systems, patterns that will emerge over time and will help to explain self-management behaviors. To help patients become better 
self-managers we have to help them address the many parts of their systems and also help them begin to act on and revise strategies based on unique, emerging patterns.

As discussed earlier, recent interventions developed to target patient-centered outcomes using systems-based theories (e.g. socio-ecological model) and concepts (e.g. motivational interviewing) have shown to be very effective. Yet, there currently exists no single, comprehensive CDSM framework to support the development of highly effective CDSM interventions. The definition presented here is an initial step in the generation of such a framework.

\section{Conclusion}

As chronic disease remains the prime health care problem in the U.S., there is a clear need to render behavioral CDSM interventions as effective as possible, and for these effects to remain stable long-term. A review of the literature indicates that most existing CDSM interventions, while effective in the short-term, may need to be revised to allow for longerlasting effects. In this hybrid concept analysis, we discovered that the majority of researchers and scholars working in the area of CDSM define this concept from the perspective of theories focused on the individual, internal characteristics of a person-selfefficacy, for example. Thus, most CDSM interventions target these individual characteristics, often times without including outside portions of a person's environment that can affect their disease status and its management. Interviews with persons currently managing chronic diseases revealed that CDSM, while involving individual characteristics, is a much more complex, fluid process involving multiple levels of a person's environment. We have put forth a definition of CDSM that is based on both literature and patient perspectives, and espouse the notion that this more comprehensive definition may help in yielding patient-centered CDSM interventions that will have longer-lasting effects.

By redefining CDSM using a patient-centric focus that recognizes the complexity of chronic disease, we can begin to develop interventions that can be adaptable to the persons' systems and prepare patients to use their existing systems to facilitate CDSM into their daily lives. Because the system is constantly in flux, CDSM interventions should be designed to also be fluid and help the patient respond better to the random interference that might from time to time impact their wellbeing. While we are not able to predict random interference, what we can do is begin to help our patients identify emerging patterns that help or hinder their management. This shift in focus to emergent pattern identification is a more patient-centered approach that moves beyond just developing problem solving skills, it also helps to better understand the forces of nature that lend to the ebb and flow of chronic disease and then how to evolve and adapt to improve quality of life.

Current findings demonstrate the need for the development of CDSM interventions that involve multiple levels of patients' environments, thereby making them and their effects more sustainable. We suggest that there is a need for pilot work in the development of CDSM interventions based on theoretical perspectives that acknowledge the complexities of a person's life. Before such pilot work can be implemented, the definition of CDSM that has been generated as a result of this hybrid concept analysis must be tested against the 
experiences of CDSM in populations outside of epilepsy. We endeavor to complete this work, and may refine the definition of CDSM based on those results.

\section{Acknowledgments}

Sources of Funding: F31NR012114 and KL2TR000163, both from the National Institutes of Health.

\section{References}

Ajzen, E. From intentions to action: A theory of planned behavior.. In: Kuhl, J.; Beckman, editors. Action Control from Cognition to Behavior. Springer-Verlag; Berlin: 1985. p. 11-39.ISBN: 978-3-642-69748-7

Bandura, A. Self-efficacy: The exercise of control. W.H. Freeman; New York: 1997.

Bandura, A. Social foundations of thought and action: A social cognitive theory. Prentice Hall; Englewood Cliff, NJ: 1986.

Barlow J, Sturt J, Hearnshaw H. Self-management interventions for people with chronic conditions in primary care: Examples from arthritis, asthma and diabetes. Health Education Journal. 2002; 61(4): 365-378.

Barlow J, Turner A, Swabyl L, Gilchristl M, Wright C, Doherty M. An 8-yr follow-up of Arthritis Self-Management Programme participants. Rheumatology. 2008; 48(2):128-133. [PubMed: 19036778]

Bodenheimer J, Wagner EH, Grumbach K. Improving primary care for patients with chronic illness. Journal of the American Medical Association. 2002; 288(14):1775-1779. [PubMed: 12365965]

Bourbeau J, Nault D, Dang-Tan T. Self-management and behaviour modification in COPD. Patient education and counseling. 2004; 52(3):271-277. [PubMed: 14998597]

Caplin D, Creer T. A self-management program for adults with asthma: Maintenance and relapse of skills. Journal of Asthma. 2001; 38(4):343-356. [PubMed: 11456388]

Clark N. Management of chronic disease by patients. Annual Review of Public Health. 2003; 24(1): 289-313. DOI 10.1146/annurev.publhealth.24.100901.141021.

Centers for Disease Control and Prevention. [February 2, 2014] Chronic diseases and health promotion. 2013. from http://www.cdc.gov/chronicdisease/overview/index.htm

Colaizzi, P. Psychological research as the phenomenologist views it.. In: Valle, R.; King, M., editors. Existential- Phenomenological Alternatives for Psychology. Oxford University Press; London: 1978. p. 48-71.

Corbin, J.; Strauss, A. Unending work and care: Managing chronic illness at home. Jossey-Bass; San Francisco, CA: 1988.

Coster S, Norman I. Cochrane reviews of educational and self-management interventions to guide nursing practice: A review. International Journal of Nursing Studies. 2009; 46(4):508-528. [PubMed: 19012889]

Fulton, JS.; Baird, C. Individual as client. In Foundations of Clinical Nurse Specialist practice. Springer; New York, NY: 2014. p. 199

Hibbard JH, Stockard J, Mahoney ER, Tusler M. Development of the patient activation measure (PAM): Conceptualizing and measuring activation in patients and consumers. Health Services Research. 2004; 39(4):1005-1026. DOI: 10.1111/j.1475-6773.2004.00269.x. [PubMed: 15230939]

Holman H, Lorig K. Patient self-management: A key to effectiveness and efficiency in care of chronic disease. Public Health Reports. 2004; 119(3):239-243. [PubMed: 15158102]

Kaplan, RM.; Anderson, JP. The General Health Policy Model: An Integrated Approach.. In: Spilker, B., editor. Quality of Life Assessments in Clinical Trials. Raven Press, Ltd.; New York: 1990.

Leventhal H, Diefenbach M, Leventhal EA. Illness cognition: Using common sense to understand treatment adherence and affect cognition interactions. Cognition and Therapy and Research. 1992; 16:143-163.

Lewin, K. Field Theory in Social Science. Harper \& Row; New York: 1951. 
Lorig K. Self-management education: More than a nice extra. Medical Care. 2003; 41(6):699-701. [PubMed: 12773834]

Lorig K, Mazonson P, Holman H. Evidence suggesting that health education for self-management in patients with chronic arthritis has sustained health benefits while reducing health care costs. Arthritis and Rheumatology. 1993; 36(4):439-446.

Lorig K, Ritter P, Stewart A, Sobel D, Brown B, Bandura A, Holman H. Chronic disease selfmanagement program. 2-year health status and health care utilization outcomes. Medical Care. 2001; 39(11):1217-1223. [PubMed: 11606875]

Marks R, Allegrante J, Lorig K. A review and synthesis of research evidence for self-efficacyenhancing interventions for reducing disability: Implications for health education practice (Part I). Health Promotion Practice. 2005a; 6(37):37-43. [PubMed: 15574526]

Marks R, Allegrante J, Lorig K. A review and synthesis of research evidence for self-efficacyenhancing interventions for reducing disability: Implications for health education practice (Part II). Health Promotion Practice. 2005b; 6(148):148-156. [PubMed: 15855284]

Meyer D, Leventhal H, Guttmann M. Commonsense models of illness: the example of hypertension. Health Psychology. 1985; 4:115-135. [PubMed: 4018002]

Miller, WR.; Rollnick, S. Motivational interviewing: Preparing people for change. Gilford Press; New York: 2002.

Miller WR, Bakas T, Buelow JM. Problems, needs, and useful strategies in older adults self-managing epilepsy: Implications for patient education and future intervention programs. Epilepsy and Behavior. 2013; 5(31):25-30. [PubMed: 24317297]

Mishel MH. Uncertainty in chronic illness. Annual Review of Nursing Research. 1999; 17(1):269_ 294.

Moore SM, Borawski EA, Cuttler L, Ievers-Landis CE, Love T. IMPACT: A multi-Level family and school intervention targeting obesity in urban youth. Contemporary Clinical Trials. 2013; 36:574586. [PubMed: 24008055]

Osborn M, Smith JA. The personal experience of chronic benign lower back pain: An interpretative phenomenological analysis. British Journal of Health Psychology. 1998; 3:65-83.

Osborne R, Wilson T, Lorig K, McColl G. Does self-management lead to sustainable health benefits in people with arthritis? A 2-year transition study of 452 Australians. Journal of Rheumatology. 2007; 34(5):1-6. [PubMed: 17216670]

Prochaska JO, Velicer WF. The Transtheoretical Model of Health Behavior Change. American Journal of Health Promotion. 1997; 12(1):38-48. doi: 10.4278/0890-1171-12.1.38. [PubMed: 10170434]

Rodgers, BL.; Knafl, KA. Concept development in nursing: Foundations, techniques, and applications. Saunders; Philadelphia: 1993.

Roaldsen KS, Elfving B, Stanghelle JK, Talme T, Mattsson E. Fear-avoidance beliefs and pain as predictors for low physical activity in patients with leg ulcer. Physiotherapy Research International. 2009; 14(3):167-180. [PubMed: 19194958]

Rush B, Ogborne A. Program logic models: Expanding their role and structures for program planning and evaluation. Canadian Journal of Program Evaluation. 1991; 6:95-106.

Ryan P, Sawin K. The individual and family self-management theory: Background and perspectives on context, process, and outcomes. Nursing Outlook. 2009; 57(4):217-225. [PubMed: 19631064]

Sandelowski M. Qualitative analysis: What it is and how to begin. Research in Nursing and Health. 1995; 18(4):371-375. [PubMed: 7624531]

Unger W, Buelow J. Hybrid concept analysis of adults newly diagnosed with epilepsy. Epilepsy \& Behavior. 2009; 14(1):89-95. [PubMed: 18796337]

Wallston KA, Wallston BA, DeVellis R. Development of the multidimensional health locus of control (MHLC) scales. Health Education \& Behavior. 1978; 6(2):160-170.

Wagner EH, Austin BT, Von Korff M. Improving outcomes in chronic illness. Managed Care Quarterly. 1996; 4(2):12-25. [PubMed: 10157259]

Whitlock EP, et al. Evaluating primary care behavioral counseling interventions: An evidence-based approach. Am J Prev Med. 2002; 22:267-284. [PubMed: 11988383] 
Zebrowitz-McArthur L, Baron RM. Toward an Ecological Theory of Social Perception. Psychological Review. 1983; 90(3):215-238. 
Table 1

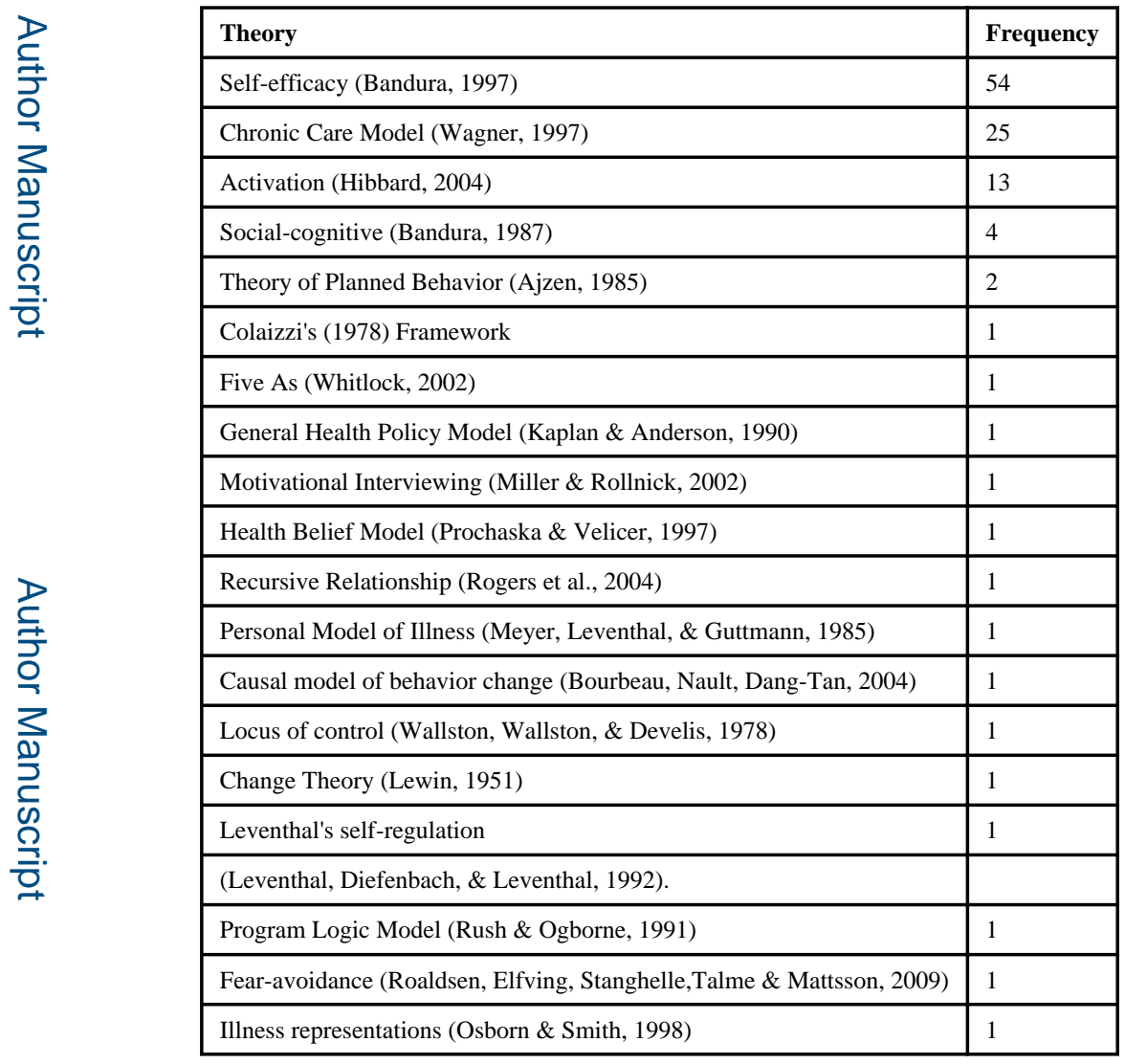




\section{Table 2}

\section{The Intrapersonal System}

a) Assessing affect and emotions - internal conditions (how I feel)

b) Assessing physical functioning - external function (my physical ability to do what I do)

c) Constant surveillance of and making use of resources (what I need to do what I do)

d) Constantly knowing that change is lurking (a real possibility)

e) Health literacy - Knowing signs and symptoms and internal and external factors that might cause an exacerbation in my chronic illness/ disease

f) Tolerating and planning for sudden or gradual onset of life interruptions

g) Incorporating treatments and side-effects so they become part of the usual and normal

Interpersonal-Selected Support System

a) Communicating with family

b) Utilizing patient-selected support persons as one aspect of self-management

Environmental Systems

Healthcare Community

a) Partnering with resource and healthcare providers to maximize daily functioning, wellness, and disease management

b) Communicating with healthcare providers 